\title{
Use of Rydberg atoms to control electron temperatures in ultracold plasmas
}

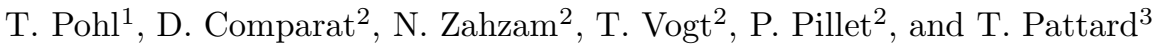 \\ 1 ITAMP, Harvard-Smithsonian Center for Astrophysics, 60 Garden Street, Cambridge, MA 02138, USA \\ 2 Laboratoire Aimé Cotton, CNRS II, Bâtiment 505, Campus d'Orsay, 91405 Orsay Cedex, France \\ 3 Max Planck Institute for the Physics of Complex Systems, Nöthnitzer Str. 38, D-01187 Dresden, Germany
}

the date of receipt and acceptance should be inserted later

\begin{abstract}
We provide a detailed theoretical and experimental study of the idea that adding Rydberg atoms to an ultracold plasma may be used as a means of controlling the electronic temperature of the plasma. We show that a certain amount of control is indeed possible, and discuss limitations for the extent of electron cooling. Experimental data are found to be in good agreement with numerical simulations.
\end{abstract}

PACS. 34.80.My Fundamental electron inelastic processes in weakly ionized gases - 32.80.Pj Optical cooling of atoms; trapping - 52.25.Kn Thermodynamics of plasmas

\section{Introduction}

Since the very first experiments creating ultracold quasineutral plasmas by photoionization of neutral atoms [1], one of the goals of this type of experiments has been to reach the regime of so-called strongly coupled plasmas. In these systems, where the Coulomb coupling parameter $\Gamma=e^{2} /\left(a k_{\mathrm{B}} T\right)$ exceeds unity (where $a$ is the average interparticle distance related to the average density $\rho$ as $a=(4 \pi \rho / 3)^{(-1 / 3)}$, and $T$ is the temperature), electrostatic Coulomb interactions between the plasma particles become important, leading to strong spatial correlations up to a crystallization of the plasma for very large $\Gamma$.

The very low temperatures characteristic of the present type of experiments suggest that ultracold plasmas should be deep within the strongly coupled regime, with Coulomb coupling parameters up to about 100 for the electrons and even up to $10^{4}$ for the ions ${ }^{1}$, being sufficiently large for Coulomb crystallization [2] to occur. However, it has been realized very quickly that various intrinsic heating mechanisms exist, which rapidly drive the system towards the weakly coupled regime. For the ions, the build-up of spatial correlations converts potential energy into kinetic energy as the system evolves from its uncorrelated initial state towards thermodynamic equilibrium and thus heats the ionic plasma component [3-8]. As a result of this disorder-induced heating, the ionic Coulomb coupling parameter decreases to a value close to unity rather than

\footnotetext{
1 Due to the large mass difference between electrons and ions, the timescale for equilibration of the whole system is very long (milliseconds) compared to the timescale of the experiments (microseconds), so that assigning independent temperatures to the electronic and ionic subsystem is well justified.
}

$10^{4}$. For the electrons, while other mechanisms such as the disorder-induced heating $[9,10]$ or threshold-lowering [11] exist, the dominant heating mechanism turns out to be three-body recombination [12], driving the electrons into the weakly coupled regime with a $\Gamma_{\mathrm{e}} \approx 0.2$.

In the meantime, several schemes have been suggested to avoid or at least weaken these heating effects, such as using fermionic atoms cooled below the Fermi temperature in the initial state [4]; an intermediate step of exciting atoms into high Rydberg states, so that the interatomic spacing is at least twice the radius of the corresponding Rydberg state [13]; using an optical lattice to create spatial correlations in the initial state $[13,14]$; or counteracting the heating by laser cooling the plasma ions [15-17]. All of these proposals are targeted at the ionic component of the plasma, hence at an increase of $\Gamma_{\mathrm{i}}$. On the other hand, a different proposal has recently been put forward aiming at the electronic plasma component [18]. The basic idea of this scheme is to influence the electronic temperature by adding, in a controlled way, additional Rydberg atoms to the plasma, which might then be collisionally ionized by the plasma electrons, thus lowering the kinetic energy of the electrons and thereby cooling them.

A first experimental study of this idea has also been reported in [18]. However, this measurement could not provide a definite answer for the feasibility of the approach, because the electronic temperature could not be determined very precisely. In this experiment, the electronic temperature has been inferred indirectly from the Kramers-Michie-King electron distribution which has been demonstrated to be correct from analogy with star cluster dynamics [19], but also by comparison with Monte Carlo [20] or particle-in-cell (PIC) [21] results. Briefly, the trap 
depth $\varphi \equiv \eta k_{\mathrm{B}} T_{\mathrm{e}}$ (with typically $\eta=5-15$ ) is strongly correlated with the size $\sigma$ of the plasma and the number $N_{\mathrm{i}}-N_{\mathrm{e}}$ of excess positive charges in the plasma $\left(N_{\mathrm{i}}\right.$ is the ion number and $N_{\mathrm{e}}$ is the number of electrons). Experimentally, we determined $N_{\mathrm{e}}$ as well as $N_{\mathrm{i}}$ at once from electron extraction. Using reasonable order-of-magnitude estimates for $\sigma$ as well as for the parameter $\eta$, it could be shown that the amount of influencing the electronic temperature by adding the Rydberg atoms was limited to a factor of less than about five. More precise statements concerning the feasibility of electron cooling, however, could not be obtained.

On the other hand, different theoretical approaches for the numerical simulation of ultracold quasineutral plasmas have been developed $[9,20,21]$, which provide detailed information about the initial plasma relaxation and the system evolution at longer timescales. In the following, we will reanalyze the experiment reported in [18] in order to characterize the degree of electron cooling achieved. By a detailed study of a broad range of initial-state parameters we demonstrate how the plasma temperature is influenced by the addition of Rydberg atoms, and how it can be manipulated by changing parameters such as the initial number of Rydberg atoms or their initial principal quantum number.

\section{Experiment}

The cesium magneto-optical trap (MOT) apparatus has been described in a previous paper [18], thus we will only briefly recall here the main aspects. Two dye laser pulses (Coumarin 500) are spatially superimposed and focused to the cold-atom cloud diameter to excite atoms initially in the $6 p_{3 / 2}$ state.

The first laser pulse, with typical energy $P_{1}=10 \mu \mathrm{J}$, creates a quasi-neutral plasma of $N_{\mathrm{i}} \approx 4 \times 10^{5}$ ions in a spherical Gaussian shape with size $\sigma \approx 150 \mu \mathrm{m}$. In reality, experiments are done with a collimated Gaussian laser with a waist smaller than the spherical Gaussian MOT, resulting in a cylindrical shape of the plasma cloud. In this sense, $\sigma \approx 150 \mu \mathrm{m}$ is an average value between the three axes. For simplicity, we assume here that the plasma can be approximated by an ionic Gaussian with spherical symmetry: $\rho_{\mathrm{i}}(r, t)=\rho_{\mathrm{i}}^{0} e^{-r^{2} /\left(2 \sigma^{2}(t)\right)}$. The case of non-spherical symmetry is nevertheless interesting and has been studied in reference [22].

The second laser pulse (ASE $<1 \%$ ) arrives with an 18 ns delay and excites typically $4 \times 10^{5}$ Rydberg atoms to the $n=24 d$ state. The Rydberg number fluctuates from pulse to pulse due to the changing overlap of the dye laser mode structure with the narrow $6 p_{3 / 2} \rightarrow$ Rydberg resonance [23]. However, the plasma created by the first laser, which is tuned just above the ionization limit, is affected only by negligible laser intensity fluctuations. The MOT gradient is turned off a few milliseconds before the laser shots. The MOT trapping lasers are turned off and a resonant $852 \mathrm{~nm}$ diode laser pulse excites the Cs $6 p_{3 / 2}$ state just before the dye laser pulse arrives. Because of the
Doppler effect, this pulse ensures that all the $6 p_{3 / 2}$ atoms are cold [23]. We have verified that $6 s$ atoms have no collisional effect on our experimental results by pushing them away using the laser light pressure just after the dye laser pulse excitation. In order to study the evolution of the cloud, we applied, after a variable time $t_{1}=0-20 \mu \mathrm{s}$, a voltage pulse large enough to field-ionize Rydberg states, leading to a signal on the MCP that is monitored using a box-car integrator. The ratio $R(t)=N_{\mathrm{R}}(t) / N_{\mathrm{R}}(t=0)$ of the number of Rydberg atoms present at time $t$ to the number of atoms at $t=0$ has been measured at $t=1 \mu$ s for various Rydberg initial principal quantum numbers $n_{0}$ and ion numbers $N_{\mathrm{i}}$. The number of Rydberg atoms present in the system after one microsecond, $N_{\mathrm{R}}(t=1 \mu \mathrm{s})$, has been determined by standard field ionization techniques allowing us to ionize all Rydberg states down to $n^{*} \approx 20$ (where $n^{*}=\sqrt{2 E_{\mathrm{b}}}$ is the effective quantum number). Some results (all data were taken at the same day) are plotted in figure 1 . The results are not sensitive to the angular momentum distribution of the electron in the Rydberg atoms, i.e. results are found to be similar for $s$ and $d$ states despite the difference in laser excitation. Error bars are not shown but are typically of the order of 10 percent, mainly due to the frequency comb fluctuation of the laser creating the Rydberg atoms. However, the difficulty to achieve perfect overlap between the two lasers leads to much larger fluctuations from day to day.

\section{Theoretical description}

The dynamics of the atoms and ions can be described accurately using a particle-in-cell treatment, where the different species are represented by testparticles which evolve under the influence of the electronic and ionic mean-field forces $[20,21]$. On the other hand, the much lighter electrons are assumed to be in a dynamically changing quasiequilibrium which we describe by a Michie-King distribution as mentioned above [21]. At each time step, the electron density is obtained by self-consistently solving the corresponding Poisson equation together with the energy conservation relation for the total system, which in addition yields the temperature of the electrons. Finally, we account for electron-Rydberg atom collisions and threebody recombination within a standard Monte Carlo procedure employing the collision rates of [24].

The initial number of electrons is determined from

$$
N_{\mathrm{i}}-N_{\mathrm{e}} \approx \sqrt{N_{\mathrm{i}} N_{*}}, \text { where } N_{*}=\frac{3}{2} k_{\mathrm{B}} T_{\mathrm{e}} \sigma \frac{4 \pi \epsilon_{0}}{q_{\mathrm{e}}^{2}} \sqrt{\frac{\pi}{2}},
$$

which we found [19] to yield an accurate description of previous measurements of the initial electron evaporation [1]. From our numerical simulations we find that the charge imbalance as determined by Eq.(1) does not increase significantly in time under typical conditions considered in this work. Preliminary experimental results, on the other hand, seem to indicate a small electron evaporation during the plasma expansion, which might be caused by rare 


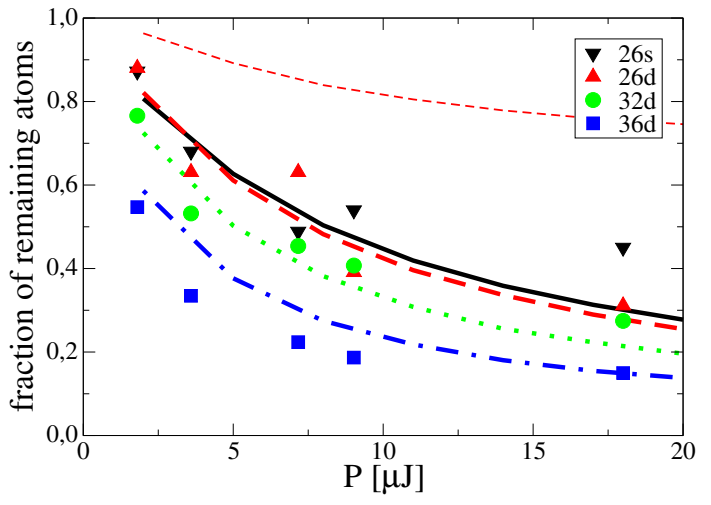

Fig. 1. (color online) Rydberg atoms present after $t=1 \mu \mathrm{s}$, normalized to the initial number $N_{\mathrm{R}}(t=0)$. Symbols: experiment for Rydberg states $26 s$ (downward triangles), 26d (upward triangles), $32 d$ (circles), $36 d$ (squares); lines: simulation for Rydberg states $26 s$ (solid), 26d (dashed), 32d (dotted), 36d (dot-dashed). The thin dashed line shows the theoretical result for the $26 d$ state, counting all atoms rather than only those with $n^{*} \geq 20$. For initial conditions, see text.

events of large energy transfer during three-body or electronRydberg atom collisions. Such evaporation processes are currently investigated but are beyond the scope of the present work.

\section{Results and discussion}

\subsection{Comparison with experiment}

In figure 1 , we compare the result of our experimental measurement of the remaining fraction of Rydberg atoms with the prediction of our theoretical model. Since the field ionization pulse can only ionize Rydberg states down to a minimum quantum number $n^{*} \approx 20$, we plot the calculated fraction of Rydberg atoms with $n^{*} \geq 20$.

Experimentally, initial-state parameters like width of the plasma cloud, absolute number of Rydberg atoms etc. are not easily controlled quantitatively. As stated in section 2, e.g., the number of Rydberg atoms generated varies from shot to shot due to the changing overlap of the dye laser mode with the Rydberg excitation resonance. For the numerical simulations shown in figure 1 , we have used the typical values $\sigma(t=0)=155 \mu \mathrm{m}, T_{\mathrm{e}}(t=0)=50 \mathrm{~K}$, and $T_{\mathrm{i}}(t=0)=100 \mu \mathrm{K}$. The initial atomic binding energy is determined from the effective quantum number $n_{0}^{*}=n_{0}-$ $\delta_{l}$, with the quantum defects $\delta_{s}=4.0$ and $\delta_{d}=2.5$. The number of plasma ions is proportional to the energy of the ionizing laser pulse, assuming a number of $N_{\mathrm{i}}=4 \times 10^{5}$ at $P_{1}=10 \mu \mathrm{J}$. The number of Rydberg atoms varies with $n_{0}$ and is determined experimentally as $N_{\mathrm{R}}(26 s)=265000$, $N_{\mathrm{R}}(26 d)=339000, N_{\mathrm{R}}(32 d)=434000$ and $N_{\mathrm{R}}(36 d)=$ 366000 . The rms-width $\sigma$ of the initial plasma cloud and the cloud of Rydberg atoms excited by the second dye laser pulse has been assumed to be equal, generating the same Gaussian density profile for ions and atoms, respectively, $\rho_{\mathrm{i} / \mathrm{R}} \equiv N_{\mathrm{i} / \mathrm{R}} \exp \left(-r^{2} /\left(2 \sigma^{2}\right)\right) /\left(2 \pi \sigma^{2}\right)^{3 / 2}$. As can be

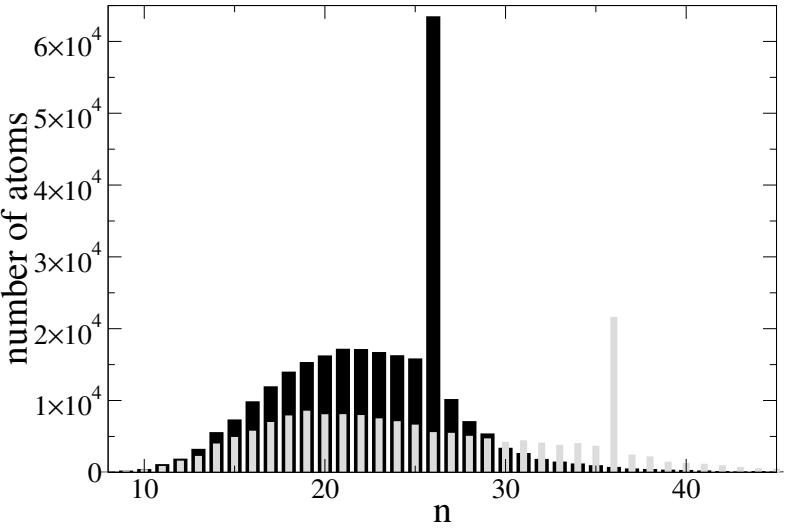

Fig. 2. Population of bound Rydberg states after $t=1 \mu \mathrm{s}$ for $P_{1}=11 \mu \mathrm{J}$ and two different initial Rydberg states $26 d$ (black bars) and 36d (grey bars).

seen, there is a good qualitative agreement between the measured data and the results of the numerical simulations. The remaining discrepancies can partly be attributed to slightly changing initial conditions in the experiment, as discussed above, on the one hand, and the difficulty of determining $R(t)$ experimentally on the other. Hence, the favorable comparison between experimental and theoretical data gives us confidence both in the validity of the numerical method as well as the experimental scheme of extracting $N_{\mathrm{R}}(t)$.

Still, we have not been able to theoretically reproduce the experimentally observed behavior for $n_{0}=22$, where practically no ionization was seen [18]. This discrepancy is even more puzzling in view of the fact that the simulations predict a large fraction of the apparent atom loss to be due to deexcitation below the field ionization limit of $n^{*}=20$. This is demonstrated in figure 2 , where we show the atomic level distribution for two different initial Rydberg states $36 d$ and $26 d$. As can be seen, a significant fraction of the atoms is deexcited to states below the ionization limit. Hence, a major part of the loss of deeply bound atoms reported in [18] should be due to deexcitation rather than ionization by the plasma electrons. To further illustrate this conclusion, we also plot the total number of remaining Rydberg atoms for the $26 d$ state in figure 1 , showing that about $2 / 3$ of the observed atom loss arises from deexcitation below the field ionization limit in this case. Since deexcitation tends to heat the electrons, the total amount of cooling from additional Rydberg atoms may thus be much lower than expected in [18].

\subsection{Electron temperature dynamics}

For a systematic study of this question, we now discuss the change of the temperature evolution when varying one of the initial-state parameters while keeping the remaining ones constant. In fig. $3 \mathrm{a}$, we show results for varying initial ion number, corresponding to a varying laser pulse energy $P_{1}$, but constant Rydberg excitation to the $36 \mathrm{~d}$ state (corresponding to different scenarios along one line 

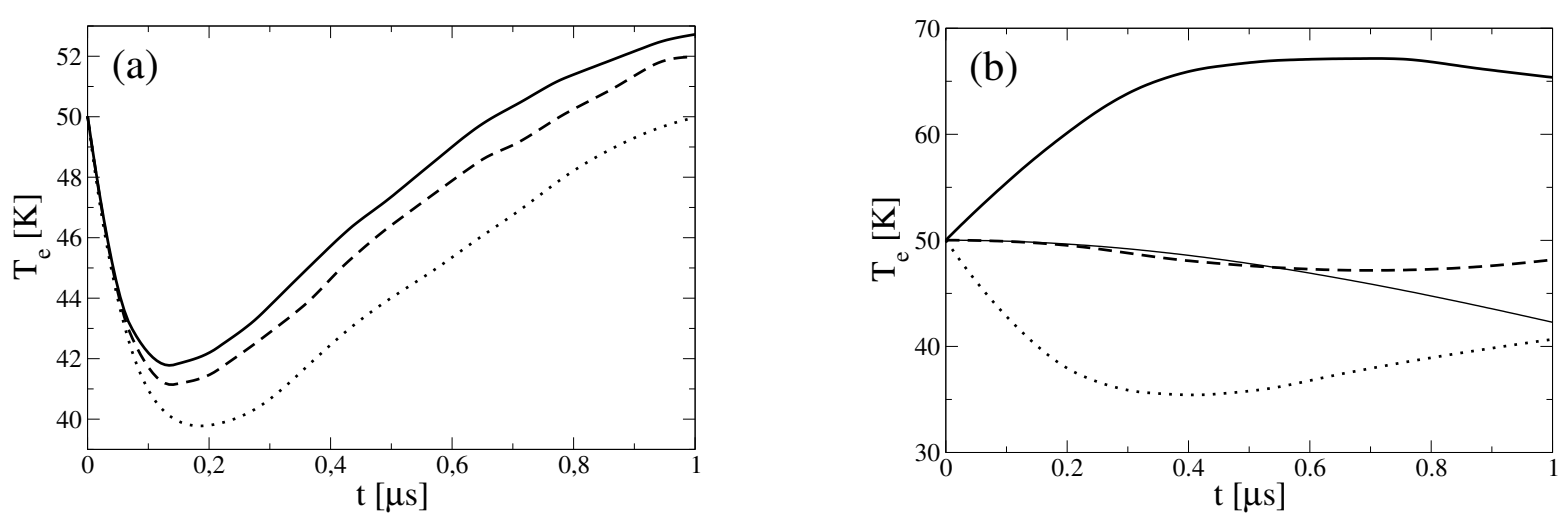

Fig. 3. Time evolution of the electronic temperature for selected initial conditions of figure 1. (a) Fixed initial excitation of $36 d$ and $N_{\mathrm{i}}=800000\left(P_{1}=20 \mu \mathrm{J}\right.$, solid line $), N_{\mathrm{i}}=680000\left(P_{1}=17 \mu \mathrm{J}\right.$, dashed line $), N_{\mathrm{i}}=440000\left(P_{1}=11 \mu \mathrm{J}\right.$, dotted line $)$. (b) Fixed $N_{\mathrm{i}}=80000\left(P_{1}=2 \mu \mathrm{J}\right)$ and $26 d$ (solid line), 31d (dashed line), 36d (dotted line). The thin solid line shows the temperature evolution for an initially pure plasma.

in fig. 1). Alternatively, we plot in $3 b$ results for different initial Rydberg excitations $n_{0}$, but constant $N_{\mathrm{i}}=8 \times 10^{4}$ (corresponding to a vertical cut at $P_{1}=2 \mu \mathrm{J}$ in fig. 1 ). Since the size of the plasma is kept constant in panel (b), we also plot the time evolution of $T_{\mathrm{e}}$ for a pure plasma, i.e. without addition of Rydberg atoms, for comparison.

As can be seen in figure $3 \mathrm{a}$, the early stage of the temperature evolution is not affected by a change of the initial electron number. Since the rate of electron-Rydberg atom collisions is linear in the number of electrons, the resulting average change of kinetic energy per electron is independent of the total number of electrons and hence the initial dynamics of the electronic temperature does not depend on the number of electrons. On the other hand, the rate of three-body recombination, which tends to heat the plasma at later stages of the system evolution, increases proportional to the third power of the charge density. Therefore, at higher plasma densities recombination sets in earlier, leading to a finally higher temperature as observed in figure 3 a.

Contrary to that, figure $3 \mathrm{~b}$ shows that a change of the initial Rydberg excitation may have dramatic consequences for the early temperature evolution. For low $n_{0}$, the plasma electrons are heated initially, while for sufficiently high $n_{0}$ a cooling effect is observed. For the $31 d$ state, corresponding to $n_{0}^{*}=28.5$, the temperature evolution is almost unaffected by the presence of the Rydberg atoms, as apparent from the close agreement with the pure plasma dynamics. This qualitative change of the plasma dynamics is connected with the existence of a kinetic bottleneck at a critical binding energy $E_{\mathrm{b}}$, below which an atom is predominantly driven to more deeply bound states. Monte-Carlo calculations [24] and recent molecular dynamics simulations [10] predict this bottleneck to be at $E_{\mathrm{b}}=4 k_{\mathrm{B}} T_{\mathrm{e}}$. For a temperature of $T_{\mathrm{e}}=$ $50 \mathrm{~K}$, this corresponds to a principal quantum number of $n^{*}=28$, in agreement with the behavior shown in figure $3 \mathrm{~b}$. Hence, precise measurements of the electron temperature evolution of the scenario under discussion would be very appealing also from this perspective, as they would allow for the first direct experimental determination of the kinetic bottleneck.

\subsection{Optimization of electron cooling}

As the discussion of the preceeding section has shown, decreasing the initial ion number extends the timescale over which electron cooling can be achieved, while increasing the initial principal quantum number increases the rate of the cooling itself. For experimental studies of a possibly strongly coupled plasma state, it is also advantageous to increase the size of the system, i.e. to decrease the plasma density, in order to stretch the timescale over which the system can be probed before the electrons ultimately heat up by three-body recombination. To illustrate this reasoning, we show in figure 4 the time evolution of the electronic Coulomb coupling parameter for a plasma of 50000 ions and a size $\sigma=600 \mu \mathrm{m}$, mixed with Rydberg atoms excited to $n^{*}=100$ whose number has been varied from $10^{5}$ to $5 \times 10^{6}$. Compared to the typical parameters of our present experiments, these values may seem somewhat exotic. However, comparable plasma sizes and excitation efficiencies are currently realized in recent ultracold plasma experiments $[6,7]$.

As can be seen in figure $4 \mathrm{a}$, the maximally achievable coupling parameter increases if more atoms are initially added to the plasma. This is due to the fact that a larger atom number leads to an increased ionization and excitation rate compared to the rate of three-body recombination, and also to a higher electron density at later times, which both increases the strength of electronic correlations. On the other hand, recombination sets in earlier for higher densities, limiting the timescale over which strong coupling effects could be observed experimentally. Further increase of the atom number therefore drives the electronic component deeper into a strongly coupled state, but also makes it more and more difficult to probe the system properties in this regime.

One possibility to circumvent this drawback might be to use more sophisticated excitation schemes, such as adding 

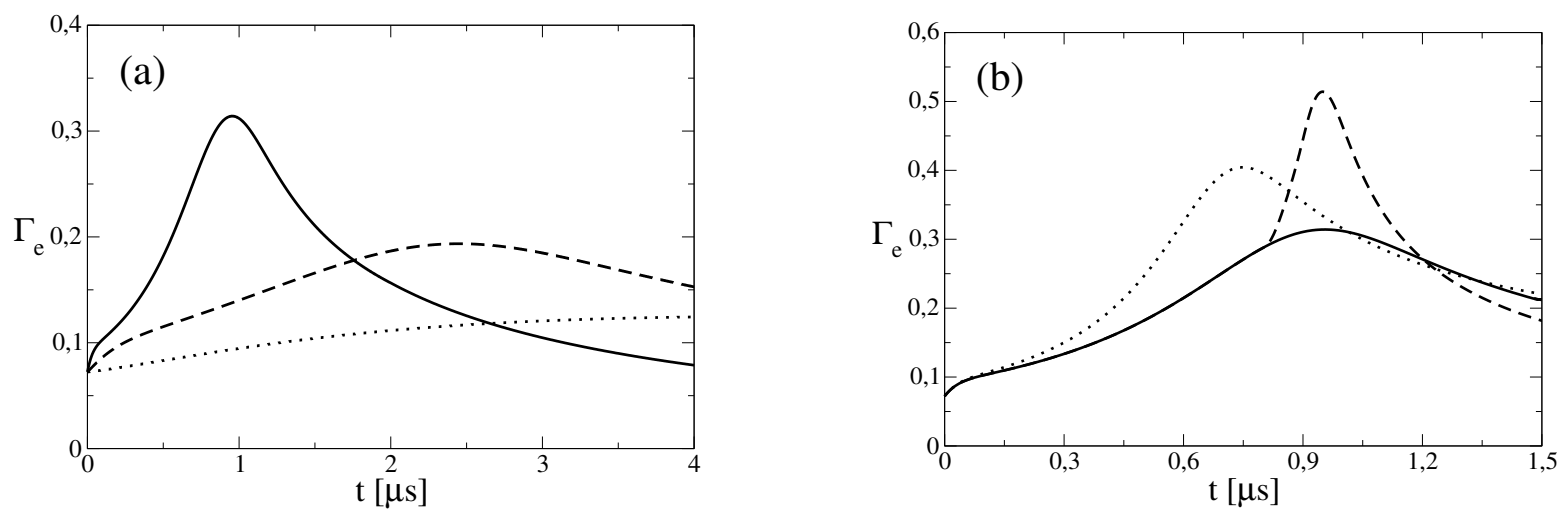

Fig. 4. Time evolution of $\Gamma_{\mathrm{e}}$ for $\sigma(0)=600 \mu \mathrm{m}, T_{\mathrm{e}}(0)=6 \mathrm{~K}, N_{\mathrm{i}}(0)=5 \times 10^{4}$. (a) $N_{\mathrm{R}}(0)=5 \times 10^{6}$ (solid line), $N_{\mathrm{R}}(0)=10^{6}$ (dashed line), $N_{\mathrm{R}}(0)=10^{5}$ (dotted line); (b) different Rydberg excitation sequences as described in the text.

further Rydberg atoms at later times rather than using a single pulse at the beginning of the plasma evolution. To test this idea, we compare in figure $4 \mathrm{~b}$ the time evolution of the Coulomb coupling parameter for the case of one single initial excitation of $5 \times 10^{6}$ Rydberg atoms (solid line) and two different, still rather simple excitation schemes. In one case, the Rydberg atom number is constantly increased by another $5 \times 10^{6}$ by a $2 \mu$ s-long excitation pulse (dotted), while in the other case the same amount is added by a short $100 \mathrm{~ns}$ pulse applied $0.8 \mu$ s after the initial Rydberg excitation (dashed). As demonstrated in figure 4b, this leads to an enhanced coupling parameter without significantly changing the timescale the system spends in the high- $\Gamma_{\mathrm{e}}$ regime.

This scenario can be further optimized by using more sophisticated pulse sequences and possibly increasing the principal quantum number in time. The latter might be necessary since the bottleneck separating net heating from net cooling, as discussed above, shifts towards higher and higher $n$ as the system cools during the plasma evolution. Investigations of such advanced schemes are certainly worthwhile. However, they are beyond the scope of the present paper.

\section{Conclusions}

In summary, we have presented a detailed study of the idea of adding Rydberg atoms to an ultracold plasma in order to control the temperature of the plasma electrons. The experiment has been performed by adding Rydberg atoms with initial principal quantum number between 20 and 40 in the center of the plasma cloud immediately after the creation of an ultracold cesium plasma with small initial temperature. The Rydberg atoms are strongly affected by the presence of the plasma, as ionization and redistribution processes occur rapidly. No qualitative differences between $s$-state and $d$-state Rydberg electrons were found.

As the numerical simulations have shown, a certain amount of control over the electron temperature is indeed possible in this way by choosing appropriate initial conditions. Changing the initial Rydberg excitation permits to control the rate and the sign of the initial temperature change while varying the initial atom number changes the timescale over which cooling can possibly be achieved.

However, the desired cooling of the plasma electrons is found to be limited to rather short times which might be challenging to resolve experimentally. Nevertheless, our simulations show that a considerable enhancement of the electronic Coulomb coupling parameter can be achieved for large atom-to-ion number ratios or more sophisticated excitation sequences.

While it turned out that our present experiments have not yet realized this regime, the optimizations proposed in this work certainly deserve further experimental studies, as they may allow for the first investigation of cold Rydberg atom dynamics in a strongly correlated electronic environment.

The theoretical results presented in this work have been limited to maximal electron Coulomb coupling parameters of about 0.5 , since the assumption of an ideal electron gas, inherent in our model, ultimately breaks down when $\Gamma_{\mathrm{e}}$ approaches unity. However, our calculations, which typically start at very low initial $\Gamma_{\mathrm{e}}$, have shown that added Rydberg atoms may drive the electrons to higher Coulomb coupling parameters, which can certainly be increased even more for more carefully chosen initial conditions to realize a strongly coupled electron component with $\Gamma_{\mathrm{e}} \approx 1$. Addressing this promising perspective for ultracold plasma studies theoretically will then require more sophisticated models to account for electronic strong coupling effects.

TPa gratefully acknowledges financial support from the ESF in the form of a short visit grant (Ref. \# 855). TPo acknowledges support by NSF through a grant for the Institute of Theoretical Atomic, Molecular and Optical Physics (ITAMP) at Harvard University and Smithsonian Astrophysical Observatory. (TP ${ }^{2}$ would like to thank J.-M. Rost for helpful discussions.

\section{References}

1. T.C. Killian, S. Kulin, S.D. Bergeson, L.A. Orozco, C. Orzel, S.L. Rolston, Phys. Rev. Lett. 83, 4776 (1999) 
2. M. Bonitz, V.S. Filinov, V.E. Fortov, P.R. Levashov and H. Fehske, Phys. Rev. Lett. 95, 235006 (2005)

3. D. Semkat,D. Kremp and M. Bonitz, Phys. Rev. E 59, 1557 (1999)

4. M.S. Murillo, Phys. Rev. Lett. 87, 115003 (2001)

5. D.O. Gericke,M.S. Murillo,D. Semkat,M. Bonitz and D. Kremp, J. Phys. A36, 6087 (2003)

6. C.E. Simien, Y.C. Chen, P. Gupta, S. Laha, Y.N. Martinez, P.G. Mickelson, S.B. Nagel, T.C. Killian, Phys. Rev. Lett. 92, 143001 (2004)

7. Y.C. Chen, C.E. Simien, S. Laha, P. Gupta, Y.N. Martinez, P.G. Mickelson, S.B. Nagel, T.C. Killian, Phys. Rev. Lett. 93, 265003 (2004)

8. E.A. Cummings, J.E. Daily, D.S. Durfee, S.D. Bergeson, Phys. Rev. Lett. 95, 235001 (2005)

9. S.G. Kuzmin, T.M. O'Neil, Phys. Rev. Lett. 88, 065003 (2002)

10. S.G. Kuzmin, T.M. O'Neil, Phys. Plasmas 9, 3743 (2002)

11. S. Kulin, T.C. Killian, S.D. Bergeson and S.L. Rolston, Phys. Rev. Lett. 85, 318 (2000)

12. F. Robicheaux, J.D. Hanson, Phys. Rev. Lett. 88, 055002 (2002)

13. D.O. Gericke, M.S. Murillo, Contrib. Plasma Phys. 43, 298 (2003)

14. T. Pohl, T. Pattard, J.M. Rost, J. Phys. B 37, L183 (2004)

15. T.C. Killian, V.S. Ashoka, P. Gupta, S. Laha, S.B. Nagel, C.E. Simien, S. Kulin, S.L. Rolston, S.D. Bergeson, J. Phys. A 36, 6077 (2003)

16. T. Pohl, T. Pattard, J.M. Rost, Phys. Rev. Lett. 92, 155003 (2004)

17. T. Pohl, T. Pattard, J.M. Rost, J. Phys. B 38, S343 (2005)

18. N. Vanhaecke, D. Comparat, D.A. Tate, P. Pillet, Phys. Rev. A 71, 013416 (2005)

19. D. Comparat, T. Vogt, N. Zahzam, M. Mudrich, P. Pillet, Mon. Not. R. Astron. Soc. 361, 1227 (2005)

20. F. Robicheaux, J.D. Hanson, Phys. Plasmas 10, 2217 (2003)

21. T. Pohl, T. Pattard, J.M. Rost, Phys. Rev. A 70, 033416 (2004)

22. E.A. Cummings, J.E. Daily, D.S. Durfee, S.D. Bergeson, Phys. Plasmas 12, 123501 (2005)

23. M.P. Robinson, B. Laburthe Tolra, M.W. Noel, T.F. Gallagher, P. Pillet, Phys. Rev. Lett. 85, 4466 (2000)

24. P. Mansbach, J. Keck, Phys. Rev. 181, 275 (1969) 